\title{
Thrombocytopenia in common variable immunodeficiency patients - clinical course, management, and effect of immunoglobulins
}

\author{
ANNA PITUCH-NOWOROLSKA ${ }^{1}$, MACIEJ SIEDLAR ${ }^{1}$, DANUTA KOWALCZYK ${ }^{1}$, \\ ANNA SZAFLARSKA², ANITA BŁAUT-SZLÓSARCZYK' ${ }^{2}$ KATARZYNA ZWONARZ² \\ ${ }^{1}$ Department of Clinical Immunology, Polish-American Institute of Paediatrics, Medical College, Jagiellonian University, Krakow, Poland \\ ${ }^{2}$ Children University Hospital, Krakow, Krakow, Poland
}

\begin{abstract}
Common variable immunodeficiency (CVID) is a primary immunodeficiency of humoral immunity with heterogeneous clinical features. Diagnosis of CVID is based on hypogammaglobulinaemia, low production of specific antibodies, and disorders of cellular immunity. The standard therapy includes replacement of specific antibodies with human immunoglobulin, prophylaxis, and symptomatic therapy of infections. High prevalence of autoimmunity is characteristic for CVID, most commonly: thrombocytopaenia and neutropaenia, celiac disease, and systemic autoimmune diseases.

The study included seven children diagnosed with CVID and treated with immunoglobulin substitution from 2 to 12 years. Thrombocytopenia was diagnosed prior to CVID in four children, developed during immunoglobulin substitution in three children. In one boy with CVID and thrombocytopaenia, haemolytic anaemia occurred, so a diagnosis of Evans syndrome was established. Therapy of thrombocytopaenia previous to CVID included steroids and/or immunoglobulins in high dose, and azathioprine. In children with CVID on regular immunoglobulin substitution, episodes of acute thrombocytopaenia were associated with infections and were treated with high doses of immunoglobulins and steroids. In two patients only chronic thrombocytopaenia was noted. Splenectomy was necessary in one patient because of severe course of thrombocytopaenia.

The results of the study indicated a supportive role of regular immunoglobulin substitution in patients with CVID and chronic thrombocytopaenia. However, regular substitution of immunoglobulins in CVID patients did not prevent the occurrence of autoimmune thrombocytopaenia episodes or exacerbations of chronic form. In episodes of acute thrombocytopaenia or exacerbations of chronic thrombocytopaenia, infusions of immunoglobulins in high dose are effective, despite previous regular substitution in the replacing dose.
\end{abstract}

Key words: children, CVID, thrombocytopaenia, immunoglobulins substitution, steroids.

(Centr Eur J Immunol 2015; 40 (1): 83-90)

\section{Introduction}

The common variable immunodeficiency (CVID) is a primary immunodeficiency of humoral immunity with heterogeneous clinical symptoms. The clinical symptoms typical for CVID are frequent, recurrent infections with poor response to therapy. The laboratory tests showed hypogammaglobulinaemia (IgG only, IgG plus IgA and/or $\operatorname{IgM})$, low production of specific antibodies in response to vaccines, and disorders of cellular immunity. The typical therapy for CVID includes the replacement of specific antibodies with human immunoglobulin substitution in intravenous (IVIG) or subcutaneous form (SCIG), standard dose $0.4-0.6 \mathrm{~g} / \mathrm{kg}$ body weight $/$ month, prophylaxis, and symptomatic therapy of infections [1-8]. Common variable immunodeficiency is associated with autoimmunity in about $30 \%$ of patients. Most common of all autoimmune diseases in CVID are cytopaenias, including thrombocytopaenia. In children, gastrointestinal autoimmune diseases (e.g. celiac disease) are noted very often, while systemic autoimmune diseases, including rheumatoid arthritis, are observed in adults [3-6, 8-10]. In half of patients with CVID and autoimmune diseases, especially cytopaenias, this autoimmunity occurs prior to diagnosis of immunodeficiency $[6,7,10]$.

Correspondence: Anna Pituch-Noworolska, Department of Clinical Immunology, Polish-American Institute of Paediatrics, Jagiellonian University, Medical College, Wielicka St. 265, 30-663 Kraków, Poland, phone/fax +48 12658 17 56, e-mail: mipituch@cyf-kr.edu.pl 
The cause of prevalence and pathomechanisms of autoimmunity in primary humoral immune deficiency, e.g. hyper IgM syndrome, X-linked agammaglobulinaemia, IgA deficiency, and CVID, are not well understood and different hypotheses have been suggested [4, 6, 8-10]. Moreover, the differences of autoimmune disease profiles between children and adults, and the different types of autoimmune diseases in a given humoral immune deficiency, are open questions. The association between total anterior circulation infarct (TACI) deficiency and higher frequency of autoimmune disease in CVID suggest the role of this particular genetic mutation in the autoimmune process. One of the possible explanations is that the partial TACI signal in the heterogeneous state may support the survival of autoreactive B cells. However, autoimmune diseases are noted in CVID patients without TACI deficiency [4, 8]. The common observation of reduced number of switched memory B cells (CD27+) and expansion of activated CD$21^{\text {low }} \mathrm{B}$ cells containing a high proportion of autoreactive $\mathrm{B}$ cells are connected with another mechanism leading to autoimmunity [4, 6, 8]. Moreover, the survival of autoreactive $B$ cells is supported by $B$ cell activating factor (BAFF) and acidic protein rich in leucine (APRIL) noted at high levels in CVID patients with autoimmunity [4, 8]. Besides the disturbances in B cell maturation and activation, $\mathrm{T}$ lymphocytes are also involved in the development of autoimmunity in CVID. The decrease of T regulatory number (CD4+, CD25+, Foxp3+) is indicated as factor facilitating the autoimmune process $[4,6,8,9]$. In the subgroup of CVID patients with lymphopaenia, an increased level of interleukin-7 (IL-7) was found, and the role of this cytokine in the expansion of autoreactive $\mathrm{T}$ cell clones was included into the mechanisms of autoimmunity in these patients [8].

Standard therapy of acute and chronic thrombocytopaenia in CVID patients is based on steroids in high doses, followed prolong therapy with maintenance doses and immunoglobulins. The intravenous infusion of high doses of immunoglobulins (1.0-2.0 g/kg body weight) are effective in increasing platelet numbers; however, a long-lasting effect is noted only in some CVID patients. This therapy with high doses of immunoglobulins in acute thrombocytopaenia episodes is more effective in patients without CVID [7, 11]. Moreover, in the clinical course of thrombocytopaenia in CVID patients, resistance to therapy is noted, suggesting active autoimmune process, despite regular immunoglobulin substitutions in replacement doses. This amount of immunoglobulins seemed to be too low to show anti-autoimmune mechanisms active when high dose was used. However, this low dose of immunoglobulins was able to stimulate dendritic cell maturation, which was shown in agammaglobulinaemia patients with defective dendritic cell phenotypes [5]. A similar defective phenotype of dendritic cells was found in CVID patients. This observation is important for the autoimmune process because dendritic cells play a critical role in predisposition to several pathological conditions, including autoimmunity. Stimulation of dendritic cell maturation might prevent the autoimmunity predisposition associated with the defective function of these cells in immunodeficient patients. It is believed that regular substitution of immunoglobulins, even in a replacing dose, restores phenotypes and normal function of dendritic cells in immunodeficiency [5]. In CVID patients with refractory thrombocytopaenia, recurrent episodes of exacerbations, or chronic thrombocytopaenia, second-line therapy with immunosuppressive drugs like azathioprine, vincristine, cyclophosphamide, and splenectomy is used $[7,10,11]$. Splenectomy is effective in these patients, similarly to patients without immunodeficiency, and safe without higher risk of severe infections in immunodeficiency [11]. The use of rituximab in CVID patients with a normal number of B cells and severe autoimmunity symptoms seemed to be another therapeutic proposition $[11,13]$, but regular substitution of immunoglobulins is indicated because of increased risk of severe infections $[4,7,11,13]$.

\section{Aim of the study}

Observations of thrombocytopaenia in patients with CVID and the effect of regular substitution of immunoglobulins in replacing dose.

\section{Material and methods}

\section{Patients}

The study included 7 patients (six boys, one girl) diagnosed as CVID (Table 1) based on hypogammaglobulinaemia (IgG, IgG/IgA, IgG/IgM), low level of specific antibodies and disturbances of cellular immunity (reverse CD4 : CD8 ratio), and poor response of lymphocytes to stimulation with mitogens (PHA, PWM) and antigens (CD3, PPD and Candida).

The clinical symptoms included recurrent infections (mainly in the respiratory tract), pneumonias, otitis, and sinusitis in older children. Following diagnosis of CVID, all patients were treated with regular immunoglobulin substitution (every month, intravenously) in a replacing dose (0.4-0.6 g/kg body weight). The thrombocytopaenia was diagnosed before diagnosis of CVID in four patients, and after CVID diagnosis in the remaining three patients (Table 2).

Thrombocytopaenia prior to diagnosis of CVID was treated with high doses of IVIG and/or steroids (high dose pulses) when the IVIG effect was short lasting. One patient underwent splenectomy followed by a high and stable number of platelets, which excluded this patient from further observation. 


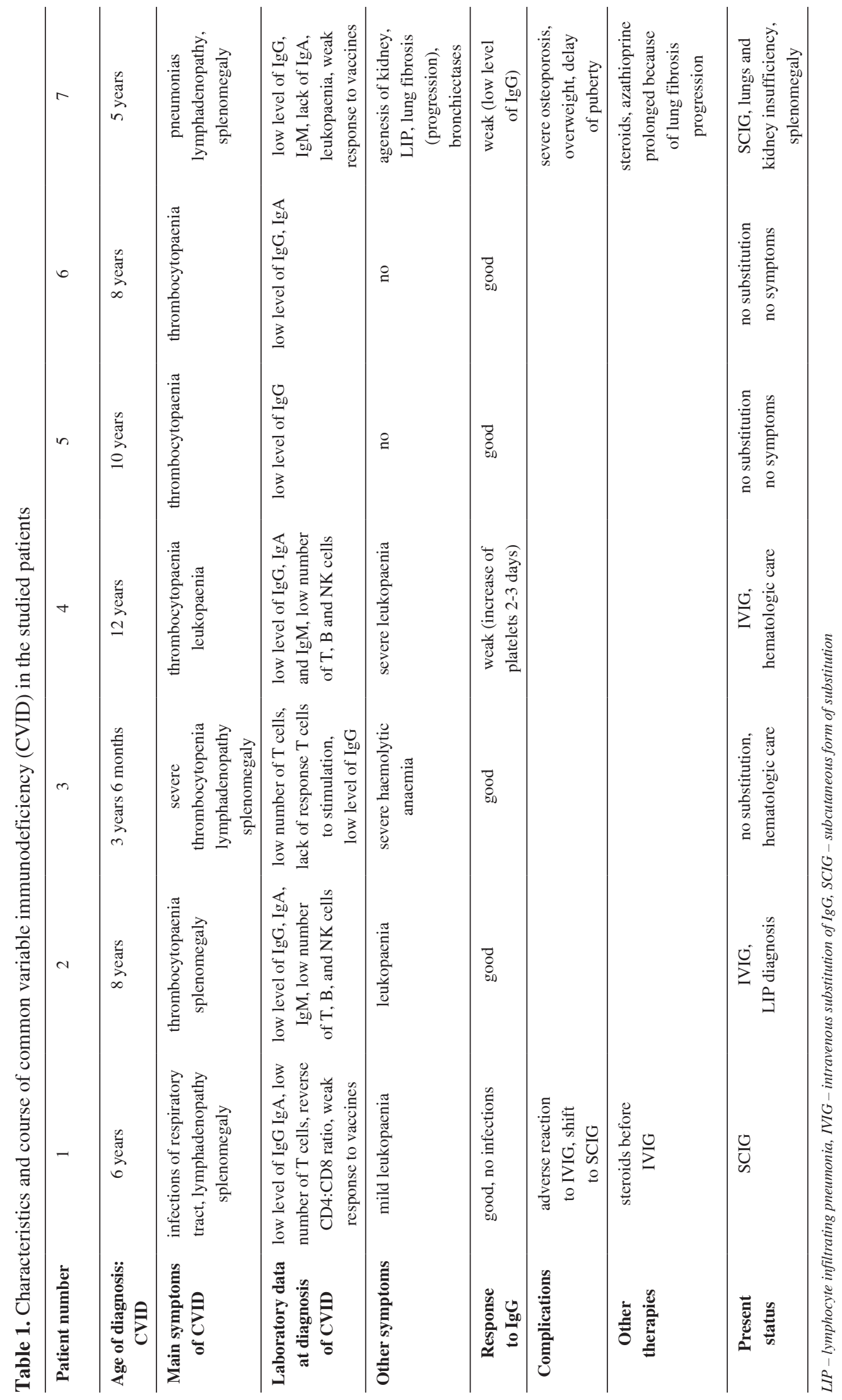




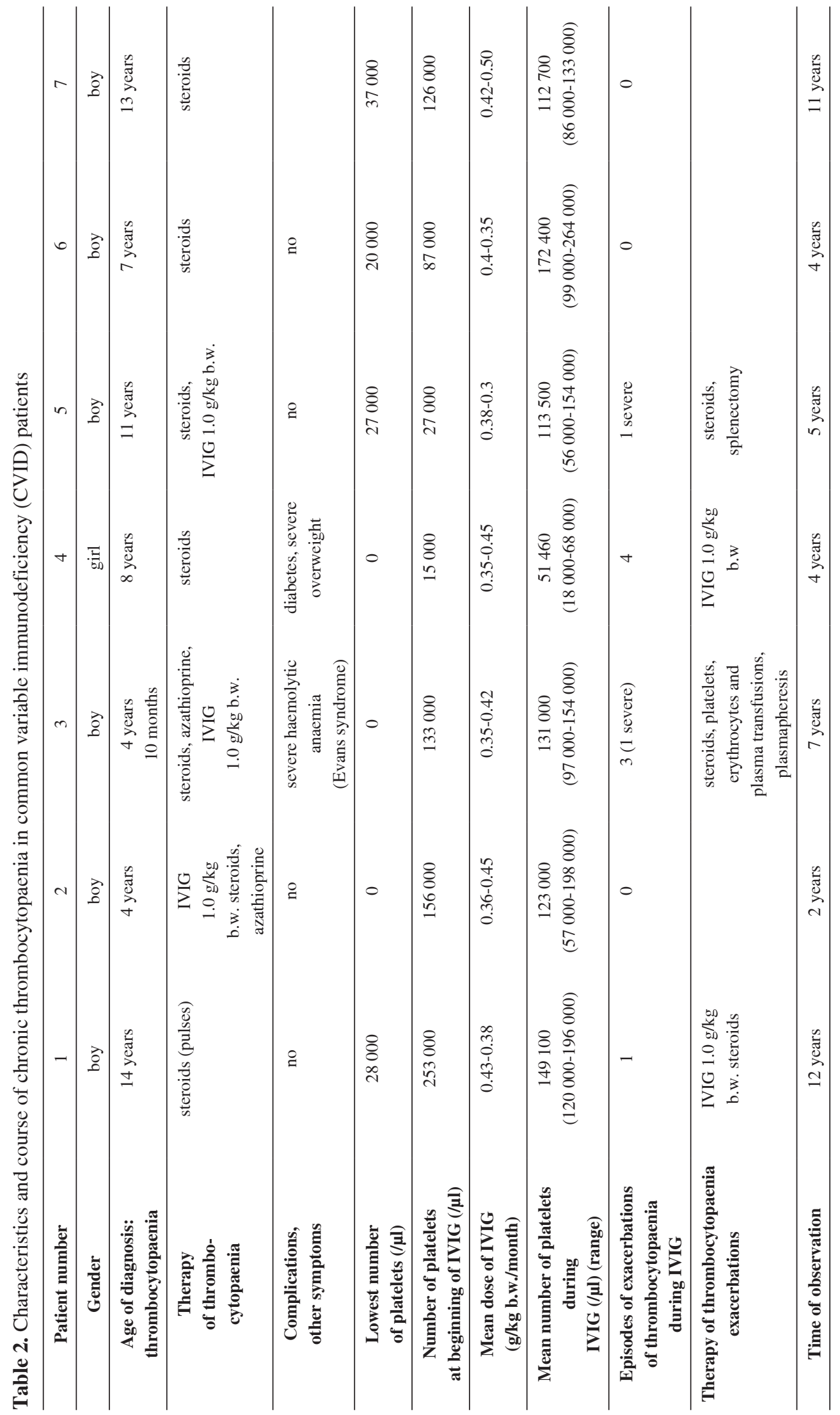




\section{Methods}

The following assays were used for patients at least twice, before the establishing the diagnosis and during the substitution of immunoglobulins. The level of immunoglobulins (IgG, $\operatorname{IgA}, \operatorname{IgM})$ in serum were assayed with nephelometry using the paediatric level of sensitivity. The percentage of T, B lymphocytes and NK cells within lymphocytes were assayed with flow cytometry followed by recalculation of the number per $\mu$ l based on total white blood cells count from the same sample. The flow cytometry assay included the $\mathrm{T}$ cell subpopulations ratio (CD4:CD8, TCR $\alpha / \beta$ : TCR $\gamma / \delta$ ), and the percentage and number of activated $\mathrm{T}$ cells (CD3/HLA-DR). The response of lymphocytes to stimulation was tested in three-day culture (PHA, CD3, and PWM) and seven-day culture (PPD, Candida) ending with $\mathrm{H}^{3}$-thymidine uptake. The borderline value of the stimulation index was 20 . The response to vaccines was assayed with anti-HBs, anti-rubeola, anti-tetanus, anti-bordetella, and anti- Streptococcus pneumoniae antibodies (if the child was vaccinated for this antigen). Moreover, the antibodies for CMV and EBV antigens were tested as common viral antigens.

The number and mean volume of platelets, and other parameters of morphology were checked on every visit associated with infusion of immunoglobulins, and in meantime if necessary. In all patients with thrombocytopaenia, the presence of autoantibodies (antinuclear standard panel with fluorescent microscopy) was assayed. The volume of platelets was normal in all of our patients (WAS syndrome exclusion). Other tests (biochemistry, microbiology, virusology, Coomb's test) and analyses were performed according to individual symptoms in patients [e.g. ultrasonography (USG), computed tomography (CT), magnetic resonance imaging (MRI), high-resolution computed tomography (HRCT) of lungs, spirometry].

\section{Results}

The onset of thrombocytopaenia in patients 1 to 6 was acute, mainly associated with infections, and the last patient (number 7) developed thrombocytopaenia in chronic form without acute episodes (Tables 1, 2).

In patient 1 thrombocytopaenia occurred after a short, mild viral infection during regular substitution of IVIG because of diagnosis of CVID [lymphadenopathy, splenomegaly, low number of $\mathrm{T}$ lymphocytes $(348 / \mu \mathrm{l})$, low number of B lymphocytes $(124 / \mu 1)]$. This patient was additionally diagnosed for ALPS (autoimmune lymphoproliferative disease), but the apoptosis was normal, so ALPS was excluded. The most probable explanation for leukopaenia and lymphopaenia despite thrombocytopaenia was spleen hyperactivity. On regular substitution in the replacing dose, the level of $\mathrm{IgG}$ was from $5.36 \mathrm{~g} / \mathrm{l}$ to $7.7 \mathrm{~g} / \mathrm{l}$, which was within the normal range for the patient's age. However, the patient developed an adverse reaction to immunoglobulin preparation. Changing the product was enough to prevent this reaction in the following infusions. The number of $\mathrm{T}$ and $\mathrm{B}$ lymphocytes was still below the lower limit of normal value for age. The episode of acute thrombocytopaenia occurred during a mild viral infection (lowest platelets number $-28000 / \mu l$ ). The effect of IVIG used in high dose $(2 \times 1.0 \mathrm{~g} / \mathrm{kg} \mathrm{b.w}$.) in this episode of thrombocytopaenia was transient, and steroids (dexamethasone pulses followed by regular oral steroid therapy) were used for four months. After this therapy the number of platelets was within the normal range. During the following four years of continued regular substitution (subcutaneous form) the number of platelets was between $120000 / \mu \mathrm{l}$ and $196000 / \mu \mathrm{l}$ without decrease, despite infections (mainly viral with mild clinical course).

Patient 2 was treated with immunoglobulins in high dose because of acute thrombocytopaenia and leukopaenia (lack of platelets and $2800 / \mu l$ of leukocytes) prior to diagnosis of CVID. The effect of steroids was short (2-3 days) despite a high dose (pulses of dexamethasone), and therapy with azathioprine was introduced. The thrombocytopaenia slowly resolved during three months. The therapy with steroid and azathioprine was stopped. After two following years without episodes of thrombocytopaenia, during a prolonged infection of the respiratory tract hypogammaglobulinaemia was noted with low number of T lymphocytes $(997 / \mu \mathrm{l})$ and NK cells $(65 / \mu \mathrm{l})$, and a lack of B lymphocytes $(20 / \mu 1)$. The splenomegaly, leukopaenia and lymphadenopathy (localised in lungs hilus), and decrease of platelet number to $57000 / \mu$ developed in the last year of observation, despite regular substitution of immunoglobulins in a dose of $0.45-0.6 \mathrm{~g} / \mathrm{kg}$ b.w. every month. Regular substitution resulted in a decreased number of infections, milder course, and better response to therapy with antibiotics. The IgG level was below $5.0 \mathrm{~g} / 1$ despite higher doses of immunoglobulins in infusions, which suggested another process of loss or process of tissue binding immunoglobulins. The additional tests for ALPS were performed because of progressing splenomegaly, lymphadenopathy, thrombocytopaenia, and leukopaenia. The number of DNT $\alpha / \beta$ lymphocytes was below $2 \%$, which excluded this diagnosis. There were no symptoms or laboratory data suggesting protein loss enteropathy. In the last month, a lung biopsy was performed due to disseminated non-inflammatory consolidations of lung tissue, implying lymphocyte interstitial pneumonia (LIP) with typical infiltrates. After high-dose steroid therapy for two weeks, followed by maintenance dose of steroids, the LIP symptoms resolved and the mean level of $\operatorname{IgG}$ increased to above $6.0 \mathrm{~g} / \mathrm{l}$.

In patient 3 low levels of IgG and IgA, low number of $\mathrm{T}$ lymphocytes, and recurrent infections were observed since 3.5 years of life, but the substitution of IgG was delayed until the $4^{\text {th }}$ year of life (overlapping THI and CVID symptoms). The response of lymphocytes to stimulation 
was poor (indexes 1-5), which suggested a diagnosis of CVID. After the episode of acute thrombocytopaenia treated with steroids, the stabilisation of platelets was obtained with azathioprine use for a prolonged period (nine months) concurrent with IVIG introduced due to established diagnosis of CVID. The number of platelets in this time was between $95000 / \mu l$ and $147000 / \mu 1$, and the dose of immunoglobulins was $0.6 \mathrm{~g} / \mathrm{kg}$ b.w. because of persistent

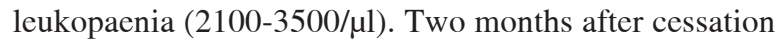
of azathioprine therapy, severe, life-threatening thrombocytopaenia (platelets nadir $-16000 / \mu \mathrm{l}$ ) and haemolytic anaemia (erythrocytes $-1.7 \mathrm{mln} / \mu \mathrm{l}$ ) with anti-erythrocyte antibodies (direct Coomb's positive test) was seen. The patient was treated with steroids (high-dose pulses), platelet and erythrocyte transfusions, and immunoglobulins in high dose $(2.0 \mathrm{~g} / \mathrm{kg}$ b.w./3 days infusion) with transient improvement of clinical symptoms. The plasmapheresis was followed by a high dose of immunoglobulins and steroids for a prolonged period (six months), which was effective. The regular substitution of immunoglobulins in a replacing dose was continued in the patient with leukopaenia, stable number of platelets (86 000-168 000/ $\mu$ ), and splenomegaly. The diagnosis was changed to Evans syndrome in CVID. Now, three years later, the patient is without substitution of immunoglobulins but under haematological control without episodes of thrombocytopaenia (platelet count 120 000-183 000/ $\mu \mathrm{l}$ ).

The first episode of thrombocytopaenia occurred in a 10-year-old girl (patient 4) after viral infection. Therapy with steroids (pulses of dexamethasone) was effective, but the number of platelets was steroid dependent, so the dose of steroids was high and used for a prolonged time. After two years of such therapy the number of platelets was within normal range, but diabetes and overweight were noted as side effects of prolonged steroid therapy. The diagnosis of CVID was made two years after the first episode of thrombocytopaenia. Hypogammaglobulinaemia (IgG and $\operatorname{IgM})$, low number of T and B lymphocytes $(820 / \mu \mathrm{l}$

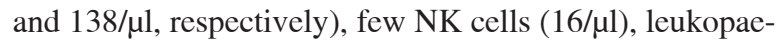
nia $(2600 / \mu \mathrm{l})$, and mainly neutropaenia were indications for regular substitution of immunoglobulins in a replacing dose. The number of platelets was checked every two weeks, and the next episode of thrombocytopaenia was noted two months after the beginning of immunoglobulin substitution. This episode of severe thrombocytopaenia (8000/ $\mu \mathrm{l}$ platelets) and the next three episodes were treated only with high doses of immunoglobulins $(2.0 \mathrm{~g} / \mathrm{kg}$ b.w./ 3 days infusion), but the effect was transient and unsatisfactory. Steroids were not used due to severe side effects after the previous therapy. The leukopaenia (number of leukocytes was from 1200 to $3800 / \mu \mathrm{l}$ ), low number of lymphocytes T (490/ $\mu \mathrm{l}), \mathrm{B}(105 / \mu \mathrm{l})$, and NK cells $(98 / \mu \mathrm{l})$, and chronic thrombocytopaenia (mean number of platelets was about $50000 / \mu \mathrm{l}$ ) were noted during the next four years of regular substitution of immunoglobulins, despite a single episode of mild infection. Last year a high level of antibodies to thyroid peroxidase was detected followed by thyroid hormone supplementation due to subclinical hypothyreosis. The number of platelets is still below normal value without diathesis, with present obesity and normal glucose level.

Patient 5 was diagnosed with CVID before the onset of thrombocytopaenia. The first episode of thrombocytopaenia occurred despite regular immunoglobulin substitution (intravenous $0.42 \mathrm{~g} / \mathrm{kg} \mathrm{b.w.).} \mathrm{Therapy} \mathrm{included} \mathrm{only} \mathrm{ster-}$ oids, and a slow increase of platelet count was observed within two months (from $27000 / \mu l$ to $44000 / \mu l$ ). In next one and a half years, the numbers of platelets (about $120000 / \mu \mathrm{l})$ and leukocytes (3500-4100/ $\mu \mathrm{l})$ were stable, and the regular substitution of immunoglobulins in a replacing dose was continued. The mild viral infection induced severe exacerbation of thrombocytopaenia and was treated with steroids (dexamethasone in pulses). The platelet number was unstable, and after eight months of therapy with steroids and immunoglobulins in high dose $(1.0-2.0 \mathrm{~g} / \mathrm{kg}$ b.w.), the decision to perform splenectomy was made by a haematologist. After this surgery the platelet count was $409000 / \mu 1$ and the boy was excluded from further observation. Due to CVID diagnosis the substitution of immunoglobulins is continued in subcutaneous form.

Patient 6 was diagnosed with CVID after the first episode of thrombocytopaenia treated with steroids. Hypogammaglobulinaemia (IgG and $\operatorname{IgA}$ ) and infections of the respiratory tract were noted as symptoms of immunodeficiency. The number of leukocytes, lymphocytes, subpopulations of T lymphocytes, B lymphocytes, and NK cells were within normal range for age. The number of platelets was $87000 / \mu l$ at the beginning of regular substitution because of CVID. In the following three years of regular substitution of immunoglobulins, the number of platelets was stable, slowly increasing to $264000 / \mu l$, without exacerbations, despite respiratory tract infections. Stable level of immunoglobulins, few infections with mild clinical course, and good response to therapy led us to finish the regular substitution. The patient was offered immunoglobulin infusion as supportive therapy during severe infection but until now (six more years of observation) he has not required it.

Patient 7 was diagnosed with CVID in the fifth year of life after hypogammaglobulinaemia during first four years of life, diagnosed as transient hypogammaglobulinaemia of infancy (THI). Infusions of immunoglobulins were indicated as supportive therapy for episodes of pneumonia, but there were no indications for regular substitution due to THI. The clinical symptoms of CVID at diagnosis included lymphadenopathy, splenomegaly (“megaspleen”), hypogammaglobulinaemia (IgG, lack of $\operatorname{IgA}$ ), and leukopaenia with low number of $\mathrm{T}$ and $\mathrm{B}$ lymphocytes in this patient. Moreover, this boy was born with one kidney. The regular substitution of immunoglobulins introduced after CVID diagnosis helped decrease the number of respira- 
tory tract infections. In gteh following years bronchiectases and progressing fibrosis with lymphocyte infiltration (lymphocytic interstitial pneumonia - LIP) developed. After two years of immunosuppressive therapy with steroids to inhibit the progress of lung fibrosis and lymphocytic infiltrates, severe adverse reactions to steroids (osteoporosis, overweight, inhibition of growth) were observed without any visible inhibitory effect on the fibrotic process. The positive effect of steroid therapy was resolving of LIP symptoms (checked with lung biopsy). The number of platelets and leukocytes were stable, close to the lower limit of normal value. The next therapy with azathioprine was administered in the hope of inhibiting the progress of lung fibrosis, but the response to this therapy was poor. Moreover, as a side effect of this immunosuppression, the number of platelets dropped below 100 000/ $\mu$ l and leukopaenia with low number of $\mathrm{T}$ and $\mathrm{B}$ lymphocytes, and lack of NK cells were noted within one year. The fibrosis of lungs is progressing slowly, but kidney insufficiency is now a clinical problem. The therapy with azathioprine was stopped. The number of platelets is still below $100000 / \mu 1$, leukocytes below 3000/ $\mu \mathrm{l}$, but the bone marrow is normal with rich cellularity and proper representation of myeloid and lymphoid ontogeny. The persistent leukopaenia, mild thrombocytopaenia, megaspleen, and lung fibrosis are still indications for suppression, but the kidney insufficiency (progressing) and severe side effect are limitations for such therapy. Now, the patient is under careful clinical control with symptomatic and supportive therapy for lung function and regular substitution of immunoglobulins (up to $0.5 \mathrm{~g} /$ $\mathrm{kg}$ b.w. subcutaneously). The prognosis for this patient is poor due to clinical symptoms and increasing laboratory parameters of lung and kidney insufficiency.

\section{Discussion}

Thrombocytopaenia associated with immunodeficiency presented two forms - episodes of acute thrombocytopaenia, alone or as a part of Evans syndrome with remission (normal number of platelets), and chronic thrombocytopaenia with platelet count always below normal level [7, 11]. In our group of CVID patients with thrombocytopaenia these (recurrent acute or chronic) forms of thrombocytopaenia were noted without relation to time of CVID diagnosis. Regular intravenous substitution of immunoglobulins in the CVID patients for exacerbation of chronic thrombocytopaenia or episodes of acute thrombocytopaenia were not effective. Routine therapy with steroids and high doses of immunoglobulins was necessary and was used with success despite regular immunoglobulin substitutions before the episode. After infusion of immunoglobulins in high dose, in remission of thrombocytopaenia, the regular substitution was continued. However, there are problems of thrombocytopaenia in CVID - short effect of high-dose immunoglobulin therapy and indication for maintenance e.g. steroid therapy to support the remission, or chronic thrombocytopaenia after short remission with normal number of platelets. If the therapy with high-dose immunoglobulins and/or steroids is effective, the remission with normal number of platelets in some CVID patients is long lasting, similarly to children without CVID. The second group of patients demonstrated chronic thrombocytopaenia with the number of platelets below 100 000/ $\mu \mathrm{l}$, and this level of platelets seemed to be stable during regular substitution of immunoglobulins in replacing doses. These patients did not require additional therapy with steroids or azathioprine as long as the number of platelets decreased and the clinical symptoms of thrombocytopaenia occurred. A large decrease in platelet count with life-threatening clinical symptoms or refractory, severe thrombocytopaenia are always indications for splenectomy without regard to CVID diagnosis $[7,10,11]$. Moreover, in CVID patients the refractory thrombocytopaenia seemed to be more frequent with a higher ratio of indications for splenectomy than in otherwise healthy children. In chronic thrombocytopaenia regular substitution of immunoglobulins in the replacing dose seemed to stabilise the platelets count, particularly in intravenous form of substitution. Clinical observations of our two patients with chronic thrombocytopaenia showed increased platelet count only for a few days after infusion of immunoglobulins, followed by a slow decrease during the following three weeks, but the level was still higher than in acute episodes. This stable, safe level of platelets meant that the patients lived with immunoglobulin substitution without additional therapy of thrombocytopaenia. Some of these patients with CVID and thrombocytopaenia demonstrate persistent leukopaenia. The intravenous regular immunoglobulin substitution in a replacing dose and the trough level of IgG have no effect on the white blood cell count in these patients. However, this leukopaenia is mild in the majority of patients, and additional therapy is needed in singular cases only.

The autoimmunity symptoms observed in CVID patients are often overlapping, which is an indication for additional diagnosis of WAS, ALPS syndrome, or rare complications of CVID, such as GLILD (granulomatous and lymphocytes interstitial lung disease) or protein loss enteropathy due to atypical celiac or other inflammatory bowel diseases (e.g. Leśniowski-Crohn's disease). These autoimmune syndromes associated with CVID are more difficult to diagnose and require more aggressive therapy due to atypical clinical course and therapy resistance.

\section{Conclusions}

Regular infusion of immunoglobulins in replacing doses is a good supportive therapy in chronic thrombocytopaenia associated with CVID.

Regular substitution of immunoglobulins in a replacing dose does not prevent the occurrence of autoimmune 
thrombocytopaenia and exacerbations of chronic thrombocytopaenia in CVID.

In episodes of acute thrombocytopaenia or exacerbations of chronic thrombocytopaenia, infusions of immunoglobulins in high dose are effective, despite previous regular substitution of immunoglobulins in a replacing dose.

The authors declare no conflict of interest.

\section{References}

1. Ameratunga R, Woon S-T, Gillis D, et al. (2013): New diagnostic criteria for common variable immune deficiency (CVID), which may assist with decisions to treat with intravenous or subcutaneous immunoglobulin. Clin Exp Immunol 174: 203-211.

2. Bayry J, Fournier EM, Maddur MS, et al. (2011): Intravenous immunoglobulins induces proliferation and immunoglobulin synthesis from patients with common immunodeficiency. J Autoimmun 36: 9-15.

3. Gathmann B, Mahlaoui N, Gerard L, et al. (2014): Clinical picture and treatment of 2212 patients with common variable immunodeficiency. J Allergy Clin Immunol 133: 1-11.

4. Cunningham-Rundles C (2011): Autoimmunity in primary immune deficiency: taking lessons from our patients. Clin Exp Immunol 164 (Suppl 2): 6-11.

5. Kaveri SV, Maddur MS, Hadge P, et al. (2011): Intravenous immunoglobulins in immunodeficiencies: more than mere replacement therapy. Clin Exp Immunol 164: 2-5.

6. Podjasek JC, Abraham RS (2012): Autoimmune cytopenias in common variable immunodeficiency. Front Immunol 3: 189.

7. Wang J, Cunningham-Rundles C (2005): Treatment and outcome of autoimmune hematologic disease in common variable immunodeficiency (CVID). J Autoimmun 25: 57-62.

8. Warnatz K, Voll R (2012): Pathogenesis of autoimmunity in common variable immunodeficiency. Front Immunol 3: 210.

9. Notarangelo LD (2009): Primary immunodeficiencies (PIDs) presenting with cytopenias. Hematology Am Soc Hematol Educ Program: 139-144.

10. Cunningham-Rundles C (2002): Hematologic complications of primary immune deficiency. Blood Rev 16: 61-64.

11. Michel M, Chanet V, Galicier L, et al. (2004): Autoimmune thrombocytopenic purpura and common variable immunodeficiency: analysis of 21 cases and review of the literature. Medicine (Baltimore) 83: 254-263.

12. Al-Ahmad M, Al-Rasheed M, Al-Muhadi A (2010): Successful use of rituximab in refractory idiopathic thrombocytopenic purpura in a patient with common variable immunodeficiency. J Investig Allergol Clin Immunol 20: 259-262.

13. Gobert D, Busel JB, Cunningham-Rundles C, et al. (2011): Efficacy and safety of rituximab in common variable immunodeficiency-associated cytopenias: a retrospective multicentre study of 33 patients. Br J Haematol 155: 498-508. 